\title{
Histological Study of Molar Pregnancy by using some of the Special Stains
}

\author{
Tuqa Yousif Sharef $(\mathrm{PhD})^{1}$, Prshang Tawfiq $(\mathrm{MSc})^{2}$
}

and Toqa J Chkhaim(MSc) ${ }^{3}$

\begin{abstract}
\end{abstract}
Background: Molar pregnancy (hydatidiform mole HM) is an abnormal form of pregnancy in which a non-viable fertilized egg implants in the uterus and will fail to come to term. A molar pregnancy is a gestational trophoblastic disease which grows into a mass in the uterus that has swollen chorionic villi. These villi grow in clusters that resemble grapes. A molar pregnancy can develop when a fertilized egg does not contain an original maternal nucleus. The diagnosis of HM is based on its morphology. In classic cases, the abnormally large villi have a vesicular or a grape like appearance. Histologically, HM presents variable degrees of circumferential trophoblastic hyperplasia and epithelial atypia. Distinguish between complete mole (CM) and partial mole (PM) is difficult especially with early diagnosis and evacuation of molar pregnancies, however, the criteria are subjective and show considerable interobserver variability. Unfortunately in our country the histological diagnosis is depend only on routine stain $(\mathrm{H} \& \mathrm{E})$ which is not giving a clear results in some cases because of that, the study design to find the similarities and differences between the two types of molar pregnancy by using some of special stains which surely distinguish between complete and partial moles samples using immunohistochemical marker P57KIP2.

Objective: To detecting if the special stain can be helpful to distinguish between the two types of molar pregnancy also to find the accuracy of the routine diagnosis of hydatidiform mole comparing with P57 immunohistochemistry stain.

Patients and Methods: The study conducted at the maternal teaching hospital in Erbil city. Thirty cases of Molar Pregnancy diagnosed, 15 of them were complete and the others were partial mole. All the samples studied histologically using H\&E and some of special stains including: PAS stain, Congo red, and Trichrom, to describe the morphological differences between the two types of mole. Also immunohistological P57 stain was used in this study to confirm the type of mole.

Results: The study result shows the morphological variation between complete and partial hydatidiform through using the routine $\mathrm{H} \& \mathrm{E}$ stain and special stains. The complete hydatidiform mole sections showed vesicular swelling of chorionic villi and circumferential trophoblastic hyperplasia, the trophoblastic proliferation grade varying in complete samples, most samples showed grade II $(46.6 \%)$ of trophoblastic proliferation, while few of samples showed grade III (13.3\%). The cases of partial hydatidiform mole showed scattered hydropic villi with irregular scalloped villous outlines and mild trophoblastic hyperplasia, trophoblastic 
distribution elucidate that the majority $(60 \%)$ of samples show zonal distribution and high percentage $(73.3 \%)$ of focal trophoblastic proliferation. The vessels in the villous stroma of most of partial molar samples (80\%) was obviously seen also the cistern appear clearly in $73.33 \%$ of the samples and all partial molar pregnancy samples showed presence of fetal part in contrast to complete mole. The PAS stain show no different between the two types of molar pregnancy samples while the Congo red stained the partial molar samples intensely comparing with complete samples opposite to the trichrome stain which showed highly color intensity in complete than in partial samples. In this study the most important noticed results was high percentage (26.66) of positive result of P57 in complete diagnosed sample by routine way.

Conclusion: The special stains can be helpful in diagnosis of hydatidiform mole and The study of p57 immunohistochemistry stain indicate present of misread in routine diagnoses of complete hydatidiform due to mimic morphological characters.

Keywords: Molar pregnancy, Hydatidiform mole, Special stains, Complete mole, Partial mole

Corresponding Author: tuqa_yusef@yahoo.com

Received: $22^{\text {th }}$ January 2019

Accepted: $12^{\text {th }}$ March 2019

\section{Introduction}

Gestational trophoblastic disease include Molar pregnancy (partial and complete), invasive mole, choriocarcinoma and placental site tumer). The molar pregnancy or hydatidiform mole (HM) is an abnormal conception, when the trophoblast develops and form placental membranes, although little or no embryonic tissue is present [1]. Hydatidiform mole represents a pathology associated with the first trimester complication its characterized by trophoblastic hyperplasia and overgrowth of placental villi[2]. Molar pregnancy or HM is rare and its frequency varies from a geographic area to another. The incidence in the United Kingdom (UK) is approximately 1 in 1000 pregnancy[3], and [1] in every 200 to 400pregnancy in Brazilian women; this is a 5 to10 fold higher incidence than that found in the United State of America and Europe [45]. The rates in Asia much higher, it's up to one in 100 pregnancies in Indonesia [6].

Pathological and cytogenetic studies have demonstrated that molar pregnancy may be either of two distinct subtypes, complete and partial hydatidiform mole. Complete moles (CM) are usually diploid and androgenic pathologically demonstrating minimal embryonic development with hydropic chorionic villi and trophoblastic hyperplasia, while partial moles (PM) are usually paternally derived triploid conceptions in which embryonic development occurs in association with trophoblastic hyperplasia [7]. 
According to WHO recommendations, beginning with 1983, the benign hydatidiform mole (complete and partial)has been included in the larger category of the gestational trophoblastic disease (GTD)because it has been considered that it represent the initial pathology which, evolutionally, may be followed by the appearance of invasive mole and choriocarcinoma [8-9].

The diagnosis of HM is based on its morphology. In classic cases, around the 2nd trimester, the abnormally large villi have a vesicular or a grape like appearance. Histologically, HM presents variable degrees of circumferential trophoblastic hyperplasia and epithelial atypia[5-10].Partial and complete mole can be distinguished histologically depend on gross morphology; the complete moles have no identifiable embryonic or fetal tissues. Classically, the chorionic villi have diffuse trophoblastic hyperplasia and generalized swelling and the trophoblast at the implantation site has diffuse, marked atypia[11] Partial moles are identified by the variable size of chorionic villi which are scattered and irregular (scalloped villous outline) [12] i.e. The villi characterized by focal swelling and focal trophoblastic hyperplasia, focal, mild atypia of trophoblasts at the implantation site; marked villous scalloping and prominent stromal trophoblast inclusions and identifiable fetal or embryonic tissues [1113].

The distinguish between CM and PM is difficult especially with early diagnosis and evacuation of molar pregnancies, however, the criteria are subjective and show considerable inter-observer variability[14] Immunohistochemical marker P57KIP2 is used recently in the diagnosis of HM. 6-1415 This study tries to find the similarities and differences between the two types of molar pregnancy by using some of special stains after surely distinguish between complete and partial moles samples using immunohistochemical marker P57KIP2.

\section{Patients and Methods}

This study conducted at the maternal teaching hospital in Erbil city. Thirty cases of Molar Pregnancy (15 of them were partial molar and others were complete molar) used in the study. The samples selected randomly depend on statistical simple random sampling technique, from the all of molar (partial and complete) pregnancy diagnosed samples that taken in the lab of the hospital during six months. The samples were endometrial biopsies that obtained by curettage. The age of aborted women ranged between 24 and 38 years, and all the cases curettage occurred during the 1st trimester of pregnancy.

All the samples were re-diagnosed. Histological diagnoses of all cases were performed by routine sections stained with hematoxyline and eosin $(\mathrm{H} \& \mathrm{E})$ and some of special stains including: PAS stain, Congo red, and Trichrom. Immunohistochemical stain P57 was also used in this study to confirm the cases of molar pregnancy.

The bases of the study depend on a comparison between partial and complete 
molar pregnancy according to the The randomized samples includes 15 histological results of the different stains.

\section{Results}

The study involves thirty cases of randomly chosen endometrial biopsies that were obtained by curettage, and diagnosed as molar pregnancy in maternal teaching hospital in Erbil city during last six months. complete molar and 15 partial molar pregnancy blocks, that re-sectioned, stained with $\mathrm{H} \& \mathrm{E}$ and diagnosed. The main diagnostic histological features of complete molar pregnancy are absence of fetal parts. The Table (1) showed the morphological features of all complete mole cases.

Table (1): Shows the morphological features of complete molar pregnancy samples

\begin{tabular}{||l||rcc||}
\hline \hline Complete mole & Total No.15 & No & $\%$ \\
\hline \hline Trophoblastic distribution & (Circumferential type) & 15 & 100 \\
& (Zonal type) & 0 & \\
\hline \hline \multirow{3}{*}{ Trophoblastic proliferation } & Grade I & 6 & $40 \%$ \\
& Grade II & 7 & $46.6 \%$ \\
& Grade III & 2 & $13.3 \%$ \\
\hline \hline \multirow{3}{*}{ Pseudo inclusion } & Absent & 2 & $13.3 \%$ \\
& Present & 13 & $86.66 \%$ \\
& & & \\
\hline \hline \multirow{2}{*}{ vessels in the villous stroma } & Absent & 15 & $100 \%$ \\
& Present & 0 & \\
\hline \hline Fetal parts & Absent & 15 & $100 \%$ \\
& Present & 0 & \\
\hline \hline Cistern & Absent & 4 & $26.6 \%$ \\
& Present & 11 & $73.33 \%$ \\
\hline \hline
\end{tabular}

Partial mole pregnancy samples shows variance morphological features than that of complete mole as its shows in Table (2), were the partial mole characterized by presence of fetal parts, less circumferential trophoblastic distribution types (40\%) among the samples and high percentage (80\%) presence of vessels in the villous stroma. 
Table (2): Shows the morphological features of partial molar pregnancy samples.

\begin{tabular}{|l|lll|}
\hline Partial mole & Total No.15 & No & $\%$ \\
\hline \hline Trophoblastic distribution & (Circumferential type) & 6 & $40 \%$ \\
& (Zonal type) & 9 & $60 \%$ \\
\hline \hline Trophoblastic proliferation & Focal & 11 & $73.3 \%$ \\
& Diffuse & 4 & $26.6 \%$ \\
\hline \hline Pseudo inclusion & Absent & 7 & $46.66 \%$ \\
& Present & 8 & $53.3 \%$ \\
\hline \hline \multirow{2}{*}{ vessels in the villous stroma } & Absent & 3 & $20 \%$ \\
& Present & 12 & $80 \%$ \\
\hline \hline Fetal parts & Absent & 0 & \\
& Present & 15 & $100 \%$ \\
\hline \hline Cistern & Absent & 4 & $26.6 \%$ \\
& Present & 11 & $73.33 \%$ \\
\hline
\end{tabular}

The Figure (1) showed the morphological features of complete and partial molar

pregnancy using the routine stain

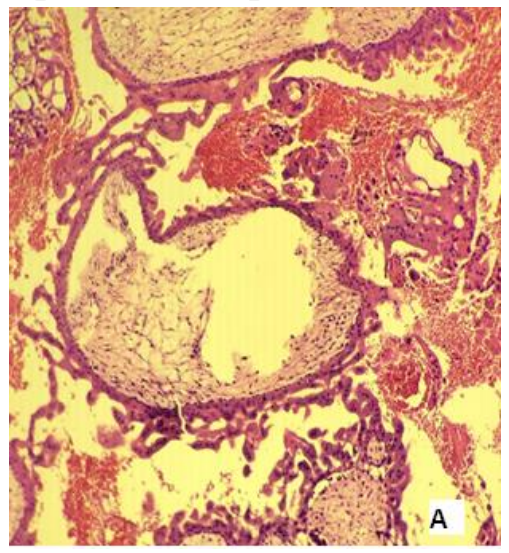
Hematoxyline and Eosin $(\mathrm{H} \& \mathrm{E})$.

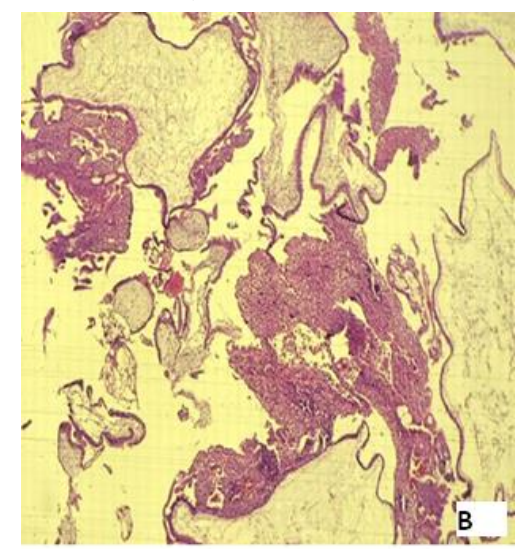

Figure (1): A-Complete Hydatidiform mole showing vesicular swelling of chorionic villi and circumferential trophoblastic hyperplasia. Trophoblastic proliferations were seen below the villus. H\&E stain x10. B-Partial mole showed hydropic villi with irregular scalloped outline and mild trophoblastic hyperplasia. H\&E stain x 10 .

The special stains also show variable intensity color between complete and partial intensity in color between complete and molar pregnancy slides, as it's showed in partial moles. In case of PAS staining Figure(2). samples there were no highly different 

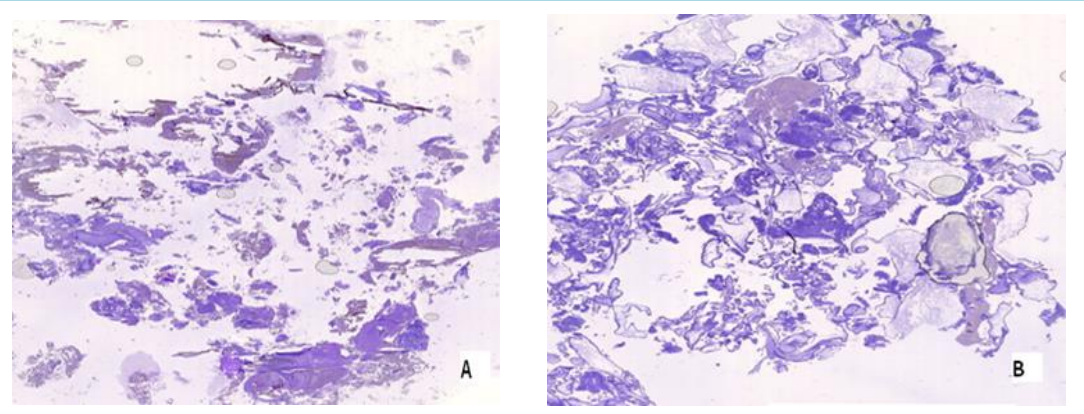

Figure (2): A- PAS stain showing moderately positive in complete mole $x$ 4. B-PAS stain showing moderately positive in partial mole $\mathrm{x} 4$.

The Congo red stain which is a special stain partial slides than that of complete one as it to detect amyloid showed highly intensity in appears in Figure(3).
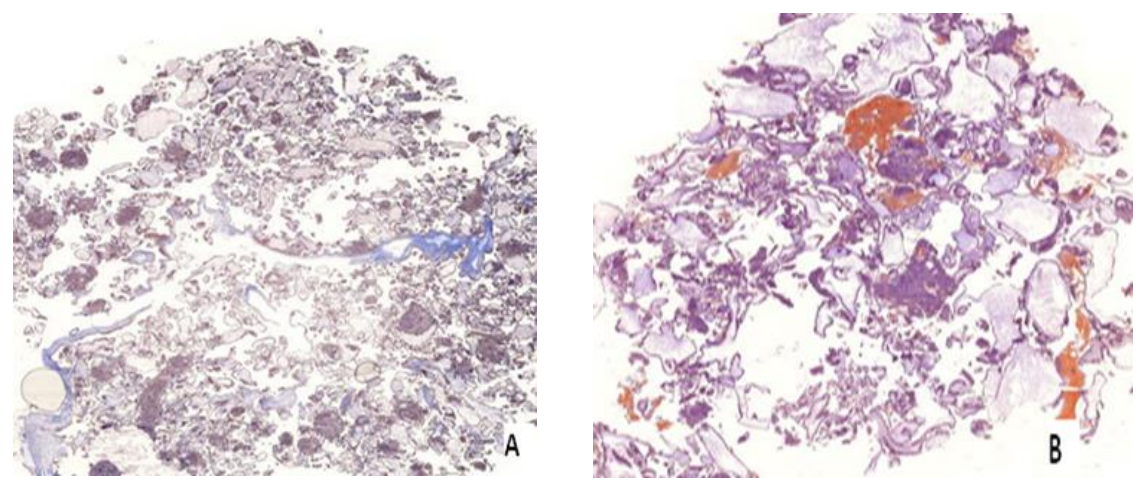

Figure (3): A- Congo red stain showing mildly positive in complete mole $\mathrm{x} 4$. B- Congo red stain showing highly positive in partial mole $\mathrm{x} 4$.

The trichrom stained samples was differed than complete mole than that in partial ones as it's the congo red. It's showed more intensity in showed in Figure (4).
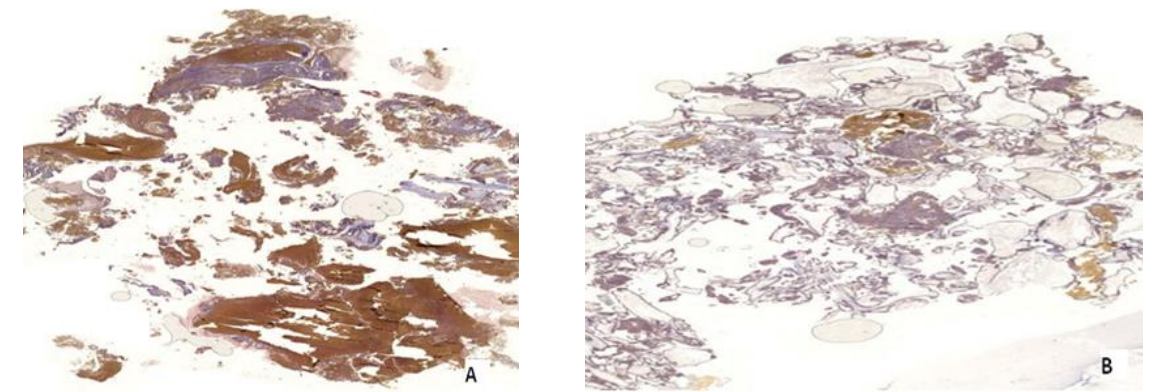

Figure (4): A-Trichrom stain showing highly positive in complete mole $\mathrm{x} 4$. B-Trichrom stains showing less positive in partial mole $\mathrm{x} 4$ comparing with that of complete mole. 
The P57 is an important complete mole.In this study the most immunohistochemistry stain used to identify important noticed results was high the partial mole from the complete mole. The percentage (26.66) of positive result of P57 P57 is positive in non-molar and partial in complete diagnosed cases as it's appear in molar pregnancy in villous stroma and Table (3). cytotrophoblast cells and negative in

Table (3): Shows the positive and negative results of molar pregnancy cases.

\begin{tabular}{|l||l|l||l|l||}
\hline \multirow{2}{*}{$\begin{array}{l}\text { Type of molar } \\
\text { pregnancy }\end{array}$} & \multicolumn{3}{|l||}{$\begin{array}{l}\text { Positive result of } \\
\text { P57 }\end{array}$} & \multicolumn{2}{l|}{$\begin{array}{l}\text { Negative results } \\
\text { of P57 }\end{array}$} \\
\cline { 2 - 5 } & No & $\%$ & No & $\%$ \\
\hline \hline $\begin{array}{l}\text { Complete mole } \\
\text { No(15) }\end{array}$ & 4 & 26.66 & 11 & 73.33 \\
\hline \hline $\begin{array}{l}\text { Partial mole } \\
(15)\end{array}$ & 15 & 100 & 0 & 0 \\
\hline
\end{tabular}

The results of P57 stain clarifies appear in molar pregnancy. figure 5 showing the positive and negative of
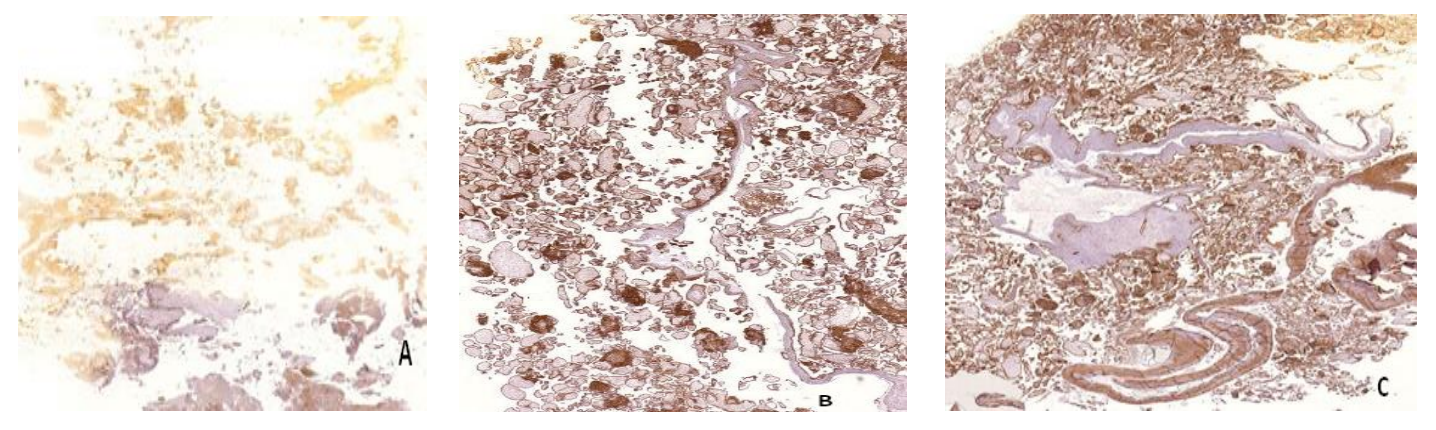

Figure (5): A-P57 marker showing negative result in complete mole x4, B- P57 marker showing positive result in complete mole $\mathrm{x} 4$. C- P57 marker showing highly positive in partial mole x4.

\section{Discussion}

The hydatidiform moles are generally subclassified into complete and partial moles. The distinction between the two types basically depend on the different morphological criteria including trophoblastic distribution, trophoblastic proliferation, Pseudo inclusion, Cistern, vessels in villous stroma and fetal parts which was the main histological features that identify complete from partial mole pregnancy[16].All partial mole sections contain fetal parts while complete type has no fetal parts [11-16].

This study demonstrated the morphological differences between the two types of molar pregnancy according to routine stain $(\mathrm{H} \& \mathrm{E})$ and special stains features sections.

The complete hydatidiform mole sections showed vesicular swelling of chorionic villi 
and circumferential trophoblastic hyperplasia as it's appear in Figure (1). The trophoblastic proliferation grade varying in complete samples, most samples showed grade II $(46.6 \%)$ of trophoblastic proliferation, while few of samples showed grade III (13.3\%) as its

mentioned in table 1and that was agree with the results of Abbas and Khafagi study [6] were the high percentage of trophoblastic proliferation grade was for grade II and less percentage samples was for grade III.

In this study all of complete samples sections showed absent of vessels in villous stroma and that was conform to the study of Jaffer and in 2011[2] and disagree with the study of Abhimanyu and RaKhee in 2017 [14].

The cases of partial hydatidiform mole showed scattered hydropic villi with irregular scalloped villous outlines and mild trophoblastic hyperplasia in the sections stained with hematoxyline and Eosin as it's appear in Figure (1) and this results was agree with many previous studies[14]. The partial hydatidiform mole examination of trophoblastic distribution elucidate that the majority $(60 \%)$ of samples show zonal distribution and high percentage $(73.3 \%)$ of focal trophoblastic proliferation as it's describe in Table (2) and that was agree with many studies [2-11]. The vessels in the villous stroma of most of partial molar samples $(80 \%)$ was obviously seen also the cistern appear clearly in $73.33 \%$ of the samples which was accordant to many studies[2-5-8-11].All partial molar pregnancy samples showed present of fetal a part which was conform by all references.

The histological study of both complete and partial hydatidiform mole using special stains showed clearly same morphological variation and differences between the two types of hydatidiform mole with little distinctness in intensity of the color of special stain.

In case of the PAS stain which was a staining method used to detect polysaccharides, mucosubstances and mucins in tissues[17], there were no different between the complete and partial hydatidiform mole samples in the reaction to this stain as its appear in the intensity color of the samples in Figure (2) and that's indicates there were no significant differences in the amount of polysaccharides , mucosubstances and mucins between complete and partial molar pregnancy.

The Congo red which is an organic compound stain used to detect amyloids in the tissue, show an interest results in this study. The partial molar samples stained intensely comparing with the complete molar cases as it's illustrated in Figures (3) and that might be indicator to high amount of amyloid in partial hydatidiform mole. In contrast to this stain, the trichrome stain which is mainly used to evaluate the type and amount of extracellular material like collagen, fibrin, muscle and elastic fiber showed high color intensity in complete molar sample comparing with partial ones which appear lighter stained in Figure (4). This result was compatible with the fact of diffused hyperplasia in complete mole[6-18]. 
The p57 immunohistochemistry stain is one of appropriated methods used to distinguish between the molar types; p57KIP2 is the protein product of the paternally imprinted but maternally expressed gene CDKN1C located on chromosome 11p15.5. Because CHM lack a maternal genomic component, they are not expected to express imprinted genes that are normally expressed by the maternal allele, and immunohistochemical analysis for p57 has been shown to be a valuable tool in the diagnosis of a CHM[619] as expected complete mole is negative for P57 while partial mole is positive in villous stroma and cytotrophoblast [5-20] but actually in this study the results was distinctive, some of the complete cases $(26.66 \%)$ showed positive results for p57 as its seen in Table (3) and demonstrate in Figure (5). The result was disagree with many of studies which confirm the efficiency of p57 in distinguish between the two types of molar pregnancy[5-14-21] but agree with the result of Abass and Al-Khafaji 2014 [6] were they find positive p57 in some of complete cases. The cases of positive p57 complete samples were most probably misread partial hydatidiform mole.

\section{Conclusions}

The complete and partial hydatidiform mole can be distinguished by routine $\mathrm{H} \& \mathrm{E}$ stain depending on histopathological parameter but the special stains can be helpful also. The trichrome special stain was highly positive in complete cases comparing to partial, while the Congo red was opposite to trichrome, where its stained intensely in partial samples comparing with the complete molar cases.

Although the histological appearance of samples, certified to diagnose the type of molar pregnancy, but the morphological mimics can cause difficulty in reliable diagnosis. The immunohistochemical stain p57 was a precisely test to distinguish between the two types of molar pregnancy. According to the results of this study, there were some of histopathological misread in the routine diagnosis of complete samples, due to morphological mimics.

The study recommended that P57 immunohistochemical stain used as a routine diagnosis for molar pregnancy in all maternity hospitals.

\section{Acknowledgment}

The researchers are grateful to the staff of maternal teaching hospital in Erbil city. specially the principal and the staff of pathological lab.

\section{Recommendation}

According to the results, the study $\begin{array}{llll}\text { recommend to use } & \text { P57 }\end{array}$ immunohistochemistry stain as a routine diagnosis for all molar pregnancy cases in all of maternity hospitals.

\section{References}

[1]Sadler T.W. Langman's Medical Embryology.10th edition, Lippincott Williams \&Wilkins USA, 2006; 51-52.

[2]Jaffar Rozina, Rahat Kalsoom and Asmaa Quershi. Histopathological review of partial and complete hydatidiform moles in a tertiary care hospital, Lahore-Pakistan. Biomedica 2011; 27:76-80. 
[3]Taylor F., D Short, R Harvey, MC Winter, J Tidy, BW Hancock, PM Savage, N Sarwar, MJ Seckl and RE Coleman. Late spontaneous resolution of persistent molar pregnancy. BJOG 2016; 123: 1175-1181. [4]Consuelo Lozoya Lopez, Vania Gloria Silami Lopes, Fabiana Rodrigues Resende, Jessica Lara Steim, Lilian Padron, Sue Yazaki Sun, Edward Araujo Junior and Antonio Braga. Gestational Trophoblastic Neoplasia after ectopic molar pregnancy: Clinical, Diagnostic and Therapeutic aspects. Rev Bras Ginecol Obstet 2018; 40: 294-299. [5]Sue Yazaki Sun, Marcia Marcelino de Souza Ishigai, Rosana Mercante Martinez, Maria Teresa de Seixas Alves and Andrea Cristina de Moraes Malinverni. Molar pregnancy: Genetic, Histological, Clinical Features and the Risk for Gestational Trophoblastic Neoplasia. J Gynecol Women`s Health 2017; 3. Dol: 10.19080/ JGWH, 2017.03.555619.

[6]Abbas Rehab K. and Khitam R. AlKhafaji. Expression of P57 Immunohistochemical Marker in Complete and Partial Hydatidiform Mole by Using Tissue Microarray Technique. IOSR- JAC 2014; 7(5): 90-95.

[7]Neil J. Sebire, Rosemary A. Fisher and Helene C. Rees. Histopathological Diagnosis of Partial and Complete Hydatidiform Mole in the first trimester of pregnancy. Pediatric and developmental pathology 2002; 6: 6977.

[8]Liliana Novac, Mihaela Niculescu, Maria Magdalena Manolea, D. Iliescu, Claudia Valentina Georgescu, Al. Comanescu, N.
Cernea and Andreea Enche. The vasculogenesis - a possible histological identification criterion for the molar pregnancy. RJME 2011; 52(1): 61-67.

[9]Berkowitz RS, Goldstein DP, The management of molar pregnancy and gestational trophoblastic tumors. In: Knapp RC, Berkowitz RS (eds), Gynecologic Oncology, 2nd edition, McGraw-Hill, New York, 1993, 328-338.

[10]Petts G, Fisher RA, Short D, Lindsay I, Seckl MJ, et al. Histopathological and immunohistochemical features of early hydatidiform mole in relation to subsequent development of persistent gestational trophoblastic disease. J Reprod Med 2014; 59: 213-220.

[11]Berkowitz Ross S. and Donald P. Goldsteir. Molar Pregnancy. N Engl Med 2009; 360: 1639-45.

[12]Sebire N.J., H. Rees, F.Paradine, M. Seckl and E. Newlands. The diagnostic implication of routine ultrasound examination in histologically confirm early molar pregnancies. Ultrasound Obstet Gynecol 2001; 18: 662-665.

[13]Montes M, Roberts D, Berkowitz RS and Genest DR. Prevalence and significance of implantation site trophoblast atypia in hydatidiform moles and in spontaneous abortions. Am J Clin Pathol 1996; 105: 4116.

[14]Abhimanyu Samadder and Rakhee Kar. Utility of P57 Immunohistochemistry in differentiating between complete mole, partial mole and non-molar or hydropic 
abortus. Indian J Med Res 2017; 145(1): 133-137.

[15]Robin D. LeGallo, MD,1 Edward B. Stelow, MD,1 et al. " Diagnosis of Hydatidiform Moles Using p57 Immunohistochemistry and HER2 Fluorescent In Situ Hybridization". Am J Clin Pathol 2008;129:749-755.

[16]Marisa R.Nucci, and Esther Oliva. Gynecologic Pathology. 1st edition. Elsevier 2009: 646-650.

[17]Carson, Freida L.; Hladik, Christa. Histotechnology: A Self-Instructional Text (3 ed.). Hong Kong: 2009 American Society for Clinical Pathology Press. pp. 137-139. ISBN 978-0-89189-581-7.

[18]Shih I-M, Mazur MT, Kurman RJ. Gestational trophoblastic disease and related lesions. In: Kurman RJ, editor. Blaustein's pathology of the female genital tract. 5th ed. New York:Springer-Verlag,2002:11931247.

[19]Robin D. LeGallo, Edward B. Stelow, and et al. " Diagnosis of Hydatidiform Moles Using p57 Immunohistochemistry and HER2 Fluorescent In Situ Hybridization". Am J Clin Pathol 2008; 129 :749-755.

[20]Vang R, Gupta M, Wu LS, Yemelyanova AV, Kurman RJ, et al. Diagnostic reproducibility of hydatidiform moles: ancillary techniques (p57 immunohistochemistry and molecular genotyping) improve morphologic diagnosis. Am J Surg Pathol 2012; 36(3): 443-453.30. [21]Sarmadi S, Izadi-Mood N, Abbasi A, Sanii S. p57KIP2 immunohistochemical expression: A useful diagnostic tool in discrimination

between

complete hydatidiform mole and its mimics. Arch Gynecol Obstet. 2011;283:743-8. 